\title{
Null infinity, H-space and equations of motion
}

\author{
Carlos Kozameh • Ezra T. Newman • \\ Gilberto Silva-Ortigoza
}

Published online: 26 September 2006

(C) Springer Science+Business Media, LLC 2006

\begin{abstract}
We discuss the existence, arising by analogy to that in algebraically special space-times, of a unique asymptotically shear-free congruence in any asymptotically flat space-time. Associated with it is a unique complex analytic curve in H-space. The surprising potential physical significance of this curve is discussed.
\end{abstract}

\section{Introduction}

In this work we describe new results concerning asymptotically flat space-times. From the asymptotic Weyl tensor obtained from an arbitrary gravitating source, we can define and construct, a 'center of mass' and 'spin-angular momentum' and their associated equations of motion by using a self-contained novel method. The basic result is that all the above information is contained in a

Fifth Award in the 2006 Essay Competition of the Gravity Research Foundation.

C. Kozameh $(\bowtie)$

FaMaF, University of Cordoba, Cordoba, Argentina

e-mail: kozameh@famaf.unc.edu.ar

E. T. Newman

Department of Physics and Astronomy, University of Pittsburgh,

Pittsburgh, PA 15260, USA

e-mail: newman@pitt.edu

G. Silva-Ortigoza

Facultad de Ciencias Físico Matemáticas, de la Universidad Autónomade Puebla, Apartado Postal 1152, Puebla, Pue., Mexico

e-mail: gsilva@fcfm.buap.mx 
complex world-line in a complex observation space with surprising physical meaning.

The starting point for this work was the observation of the important role that shear-free null geodesic congruences (ngc) played in the history of general relativity and how that role could be taken in the far more general situation of asymptotically flat space-time, by asymptotically shear-free ngc's.

One of the major theoretical advances in General Relativity of the last half century was the development of the theory of asymptotically flat solutions of the Einstein/Einstein-Maxwell equations. This culminated in the proof [1] that from the field equations, gravitational radiation could be predicted; gravitational waves carry away mass and momentum. Bondi's epic work included explicit expressions for the total energy/momentum, given as terms in the asymptotic Weyl tensor. Near this time [2] the existence of the four principal null vectors $[3,4]$ (pnv's) of the Weyl tensor was discovered with the associated existence of the algebraically special solutions (ASS) defined by degeneracy in the four pnv's. From it came the Goldberg-Sachs theorem [5]: the degenerate pnv being the tangent vector field to a ngc that is shear free. The physically important Schwarzschild, Reissner-Nordstrom, Kerr and charged Kerr, the RobinsonTrautman metrics [6], etc., lie in this class.

Recently [7-10] we returned to the study of asymptotically flat Einstein/ Einstein-Maxwell fields and discovered a hidden structure. Though shearfree ngc's cannot be found in arbitrary space-times, their idea can be generalized to asymptotically shear-free ngc's. which do exist in such space-times. The main development was the realization that for any asymptotically flat Einstein space-time (or Einstein-Maxwell with non-vanishing total electric charge) there existed a class of regular asymptotically shear-free (generally twisting) ngc's where individual members of the class are determined by the arbitrary choice of a complex analytic world-line in the complex four-dimensional $\mathrm{H}$-space. Among these congruences, one can be singled out canonically; two for the EinsteinMaxwell case. The unique choice is made, in the vacuum case, by forcing certain Weyl tensor terms to vanish-the counterpart of vanishing type II Weyl-tensors terms. For the Einstein-Maxwell fields, two uniquely defined world-lines are found: one from the asymptotic Maxwell tensor, the other again by mimicking type II Weyl-tensors. We confined ourselves to the case where the two worldlines coincided.

Our formal results appear to have a surprising physical significance. The Bondi four-momentum is determined directly in terms of the world-line while the time evolution of the four-momentum then determines the equations of motion. With no assumed models, simply from the Einstein-Maxwell equations, the real part of the world-line yields the classical equations of motion for a 'particle' with structure while the imaginary part becomes the spin per unit mass. These equations of motion include the radiation reaction term and, in addition, a mass-loss term that appears to suppress the run-away behavior. 


\section{Structures at null infinity}

As the details [7-10] of this work are complicated, we simply outline the basic ideas.

Begin with an arbitrary asymptotically flat Einstein or Einstein-Maxwell space-time. In a neighborhood of future null infinity, $\mathfrak{I}^{+}$, we introduce a Bondi coordinate system, $(u, \zeta, \bar{\zeta}, r)$, with a Bondi tetrad, $(l, n, m, \bar{m})$. At $\mathfrak{I}^{+}, l$ is tangent to the Bondi null surfaces, $n$ is tangent to the null generators of $\mathfrak{I}^{+}$while $(m, \bar{m})$ are the complex tangent vectors to the Bondi slices, $u=$ const. The asymptotic shear of the Bondi congruence, $\sigma(u, \zeta, \bar{\zeta})$, is an arbitrary complex function on $\mathfrak{I}^{+}$that is assumed to be analytic in its three arguments, extendable into the complex. The important physical quantities, the Bondi mass/momentum, are obtained as two-surface integrals of components of the Weyl tensor with, in the vacuum case, their evolution in time determined from the shear:

$$
\begin{aligned}
P_{a}\left(u_{\mathrm{B}}\right) & \equiv\left(M c,-P^{i}\right)=-\frac{c^{3}}{8 \pi G} \oint \Psi l_{a} \mathrm{~d} S, \\
\frac{\mathrm{d}}{\mathrm{d} u} P_{a}\left(u_{\mathrm{B}}\right) & =-\frac{c^{3}}{8 \pi G} \int\left(\partial_{u} \sigma \partial_{u} \bar{\sigma}\right) l_{a} \mathrm{~d} S,
\end{aligned}
$$

where $\Psi(u, \zeta, \bar{\zeta})$ is the Bondi mass aspect. A generalization for the EinsteinMaxwell case exists.

We consider a new ngc in the neighborhood of $\mathfrak{I}^{+}$whose tangent vector $l^{*}$ is given by

$$
l^{*}=l+\frac{L(u, \zeta, \bar{\zeta})}{r} \bar{m}+\frac{\bar{L}(u, \zeta, \bar{\zeta})}{r} m+O\left(r^{-2}\right) .
$$

$L$ is the complex stereographic coordinate on the sphere of past null directions of the past light-cone for any point on $\mathfrak{I}^{+}$and $L(u, \zeta, \bar{\zeta})$ is an arbitrary stereographic field on $\mathfrak{I}^{+}$. If $L(u, \zeta, \bar{\zeta})$ is chosen to satisfy the partial differential equation [11]

$$
ð L+L L,_{u}=\sigma(u, \zeta, \bar{\zeta}),
$$

the tangent vector field $l^{*}$, Eq. (2.3), determines an asymptotically shear-free ngc. (When $\sigma(u, \zeta, \bar{\zeta})=0$, solutions to (2.4) lead to twistor-space and the Kerr theorem.) A surprising aspect of (2.4) lies in the freedom in the choice of solutions:

Theorem 2.1 Solutions to Eq. (2.4) that are regular on $\mathfrak{I}^{+}$are given by the following procedure: first we note the general regular solution of the 'good cut' equation,

$$
ð^{2} Z=\sigma(Z, \zeta, \bar{\zeta}),
$$

can be written as

$$
Z=Z\left(z^{a}, \zeta, \bar{\zeta}\right)
$$


where the four complex integration parameters $z^{a}$ define H-space. From an arbitrary complex analytic world-line in $H$-space,

$$
z^{a}=\xi^{a}(\tau),
$$

the solution to (2.4) can be expressed parametrically by

$$
\begin{aligned}
L(u, \zeta, \bar{\zeta}) & =\partial X(\tau, \zeta, \bar{\zeta}), \\
u & =X(\tau, \zeta, \bar{\zeta}),
\end{aligned}
$$

with

$$
X(\tau, \zeta, \bar{\zeta}) \equiv Z\left(\xi^{a}(\tau), \zeta, \bar{\zeta}\right)
$$

In other words, the regular solutions of (2.4) are given uniquely, modulo the arbitrary choice of a complex curve in H-space.

In order to choose this line uniquely we note that for (asymptotically flat) algebraically special, vacuum metrics, there is a canonical choice of this worldline that produces not only an asymptotically shear-free ngc but a fully shear-free ngc throughout the space-time. With this canonical curve, one has a canonical $L(u, \zeta, \bar{\zeta})$ and $l^{*}$. Algebraic specialness defines the NP-tetrad form [12] of the Weyl tensor:

$$
\Psi_{0}=\Psi_{1}=0 .
$$

For general asymptotic solutions, this property can not be duplicated. Nevertheless, the freedom in the choice of the curve can be eliminated by a much weaker condition. Analogous to Eq. (2.8), we force just the $l=1$ harmonic component of the leading asymptotic term in $\Psi_{1}$ to vanish:

$$
\begin{aligned}
\Psi_{1}^{0}[l & =1]=0 . \\
\left(\Psi_{0}^{0}[l\right. & =1] \text { always vanishes })
\end{aligned}
$$

This requirement, which can always be implemented, leads to equations of motion for the determination of the curve.

We stress that the details are extremely complicated and that many approximations are needed. Also, via Eq. (2.7), the parameter $\tau$, in $\xi^{a}(\tau)$, was changed to $u$, entailing further approximations. In a canonically determined coordinate system, the curve description was decomposed into its real and imaginary parts, $\xi^{k}(\tau)=\xi_{R}^{k}(\tau)+i \xi_{I}^{k}(\tau)$.

\section{Interpretations}

From a series of arguments given below, the following physical meaning to the curve emerged: 
The parameter space, $\mathrm{H}$-space, should not to be treated as real in any senseinstead it should be viewed as an observation space; a screen space that captures certain images of the physical space. In the physical space-time there is a complicated physical system, a gravitating, charged, mass distribution, that creates curvature and an electromagnetic field that can be 'observed' only from its asymptotic behavior; we can not 'see' individual masses, charges, spins, etc., but instead only the large-scale behavior. We interpret the curve as an intrinsic complex center-of-mass and center-of-charge world-line (with equations of motion) for the entire system in this observation space.

Arguments $[9,10]$ :

1. By including Maxwell fields, we obtain (as an approximation) the relationship between the Bondi three-momentum/mass and the curve velocity and acceleration:

$$
P^{k}=M \frac{\mathrm{d} \xi_{R}^{k}}{\mathrm{~d} u}-\frac{2 q^{2}}{3 c^{3}} \frac{\mathrm{d}^{2} \xi_{R}^{k}}{\mathrm{~d} u^{2}}-\frac{3}{2 c} M \epsilon_{i j k} \frac{\mathrm{d} \xi_{I}^{i}}{\mathrm{~d} u} \frac{\mathrm{d} \xi_{R}^{j}}{\mathrm{~d} u}+\cdots
$$

The first term is trivial, the second term creates radiation reaction forces, the third resembles the Mathisson-Papapetrou spin-velocity coupling, with $M \xi_{I}^{i}$ identified as the spin vector.

2. If (3.1) is used in the Bondi mass/momentum loss equation, Eq. (2.2) yields dynamic equations for $\xi_{R}(u)$ :

$$
\begin{aligned}
M \frac{\mathrm{d}^{2} \xi_{R}^{k}}{\mathrm{~d} u^{2}}= & \frac{2 q^{2}}{3 c^{3}} \frac{\mathrm{d}^{3} \xi_{R}^{k}}{\mathrm{~d} u^{3}}-\frac{\mathrm{d} M}{\mathrm{~d} u} \frac{d \xi_{R}^{k}}{\mathrm{~d} u}+\frac{3}{2 c} M \epsilon_{i j k} \frac{\mathrm{d}}{\mathrm{d} u}\left\{\frac{\mathrm{d} \xi_{I}^{i}}{\mathrm{~d} u} \frac{\mathrm{d} \xi_{R}^{j}}{\mathrm{~d} u}\right\}+\cdots \\
& + \text { Bondi momentum loss. }
\end{aligned}
$$

In classical $\mathrm{E} \& \mathrm{M}$, using the Lorentz self-force, one finds the radiationreaction term $\frac{2 q^{2}}{3 c^{3}} \frac{\mathrm{d}^{3} \xi_{R}^{k}}{\mathrm{~d} u^{3}}$. Here, at linear order, we obtain exactly the same term without any model building or infinities. We also see there is a new term, $\frac{\mathrm{d} M}{\mathrm{~d} u} \frac{\mathrm{d} \xi_{R}^{k}}{\mathrm{~d} u}$, arising from the Bondi mass loss. In simple numerical examples, the run-away solutions from the classical radiation reaction force were damped-out by this term. This observation must be studied in more detail.

3. In the mass loss, Eq. (2.2), electric and magnetic dipole radiation appear with second derivatives of $q \xi^{k}\left(u_{\mathrm{B}}\right)=q\left(\xi_{R}^{k}+i \xi_{I}^{k}\right)$, thus identifying $q \xi_{R}^{k}$ and $q \xi_{I}^{k}$ as the electric and magnetic dipole moments. The numerical factors agree with dipole radiation derived from Maxwell theory in Minkowski space.

4. The Kerr space-time has an associated complex space and unique complex world-line with imaginary part, $\left|\xi_{I}^{k}\right|=a$, determining the spin angularmomentum, $S=M c a$. For the charged Kerr metric, the same complex world-line yields the magnetic dipole moment $\mu=q a$ in addition to the spin. (Maxwell fields $[8,13,14]$ in Minkowski space have $\mu=q \xi_{I}$.) 
It appears natural to identify $\xi_{I}^{k}$ as the imaginary center of mass and charge and define the full $\xi^{k}\left(u_{\mathrm{B}}\right)$ as the complex center of mass and center of charge. This allows a (complex) unification of many examples of electric and magnetic phenomena.

5. For the charged Kerr solution, in the gyromagnetic ratio, $S / \mu=M c / q$, the imaginary displacement drops out resulting in the Dirac value, $g=2$. It follows that for all asymptotically flat Einstein-Maxwell fields, where the two complex H-space world-lines coincide, again $g=2$.

Though there is still much to understand, this 'screen' view of these H-space curves appears to be essentially correct.

Acknowledgments This material is based upon work (partially) supported by the National Science Foundation under Grant No. PHY-0244513. Any opinions, findings, and conclusions or recommendations expressed in this material are those of the authors and do not necessarily reflect the views of the National Science Foundation. E.T.N. thanks the NSF for this support. G.S.O. acknowledges the financial support from CONACyT through Grand No.44515-F, VIEP-BUAP through Grant No.17/EXC/05 and Sistema Nacional de Investigadores (SNI-México). C.N.K. would like to thank CONICET for support.

\section{References}

1. Bondi, H., van der Burg, M.G.J., Metzner, A.W.K.: Proc. R. Soc. Lond. Ser. A 269, 21 (1962)

2. Newman, E.T., Tod, K.P.: In: Held, A. (ed.) General Relativity and Gravitation, vol. 2. Plenum (1980)

3. Penrose, R., Rindler, W.: Spinors and Space-time, vol. I. Cambridge University Press, Cambridge (1984)

4. Pirani, F.A.E.: In: Deser, S., Ford, K. (eds.) Brandeis Lectures on General Relativity. PrenticeHall, New York (1962)

5. Goldberg, J.N., Sachs, R.K.: Acta Phys. Pol. 22(Suppl), 13 (1962)

6. Robinson, I., Trautman, A.: Proc. Roy. Soc. Lond. Ser. A 289, 463 (1962)

7. Kozameh, C., Newman, E.T.: Class. Quantum Grav. 22, 4659-4665 (2005)

8. Kozameh, C., Newman, E.T.: Class. Quantum Grav. 22, 4667-4678 (2005)

9. Kozameh, C., Newman, E.T., Silva-Ortigoza, G.: Class. Quantum Grav. 22, 4679-4698 (2005)

10. Newman, E.T., Silva-Ortigoza, G.: Class. Quantum Grav. 23, 91-113 (2006)

11. Aronson, B., Newman, E.T.: J. Math. Phys. 13, 1847-1851 (1972)

12. Newman, E.T., Penrose, R.: J. Math. Phys. 3, 566 (1962)

13. Newman, E.T.: Phys.Rev. D 65, 104005 (2002)

14. Newman, E.T.: Class. Quantum Grav. 21, 1-25 (2004) 